\title{
The universality theorem for $L$-functions associated with ideal class characters
}

\author{
by \\ Hidehiko Mishou (Nagoya)
}

1. Introduction. In this paper we prove the universality theorem for $L$-functions associated with ideal class characters of algebraic number fields. First of all, we explain what is the universality theorem.

Let $s=\sigma+i t$ be a complex number and let $\zeta(s)$ be the Riemann zetafunction. It is known that $\zeta(s)$ has several arithmetic and functional properties such as the functional equation and the Euler product formula. In addition to these properties, S. M. Voronin [16] found the following remarkable result called the universality theorem for $\zeta(s)$ in 1975.

Voronin's TheOREM. Let $0<r<1 / 4$ and $f(s)$ be a continuous function on the disk $|s| \leq r$ such that $f(s) \neq 0$ on $|s| \leq r$ and $f(s)$ is holomorphic in $|s|<r$. Then for every $\varepsilon>0$, we have

$$
\liminf _{T \rightarrow \infty} \frac{m\left(\left\{\tau \in[0, T]\left|\max _{|s| \leq r}\right| \zeta(s+3 / 4+i \tau)-f(s) \mid<\varepsilon\right\}\right)}{T}>0
$$

where $m$ is the Lebesgue measure on $\mathbb{R}$.

Roughly speaking, this theorem asserts that any analytic function which satisfies the above conditions can be uniformly approximated on the disk by vertical translations of $\zeta(s)$, and the set of real numbers which give such approximation has a positive lower density.

After Voronin's work, many mathematicians studied the universality property of other zeta-functions. In 1979, S. M. Gonek [4] proved the universality theorem for Hurwitz zeta-functions $\zeta(s, \alpha)$ if $\alpha$ is rational or transcendental. In 1980, A. Reich [14] proved it for Dedekind zeta-functions.

These results were proved by the method based on Voronin's original proof. But in 1981, B. Bagchi [2] proved the limit theorem for $\zeta(s)$ which is concerned with probability measures and gave an alternative proof of the universality theorem for $\zeta(s)$. In [3], he also proved the joint universality

2000 Mathematics Subject Classification: Primary 11M41. 
theorem for Dirichlet $L$-functions, which asserts that the universality holds for several $L$-functions simultaneously.

Recently the study of the universality theorems and the limit theorems has been advanced by A. Laurinčikas and K. Matsumoto. They proved in [8] the universality theorem for zeta-functions attached to cusp forms. In [9] Matsumoto considered a certain class of $L$-functions which contains both Dedekind zeta and cusp form zeta, and Laurinčikas [7] gave sufficient conditions under which the limit theorems and the universality theorems hold for those $L$-functions. We note that $L$-functions treated in this paper are included in this class, but do not satisfy the conditions of Laurinčikas' paper.

To state our result, we recall some notions from algebraic number theory. Let $K$ be a finite extension of $\mathbb{Q}, O_{K}$ the integer ring of $K$ and $\mathfrak{f}$ an ideal of $O_{K}$.

For $\alpha \in K$, we write

$$
\alpha \equiv 1 \bmod \tilde{\mathfrak{f}}
$$

if $\alpha=a / b, a, b \in O_{K}, a-b \in \mathfrak{f}, \alpha \succ 0$.

We set

$$
A(\mathfrak{f})=\{\mathfrak{a}: \text { ideal of } K \mid(\mathfrak{a}, \mathfrak{f})=1\}
$$

and

$$
B(\widetilde{\mathfrak{f}})=\{(\alpha) \mid \alpha \in K, \alpha \equiv 1 \bmod \widetilde{\mathfrak{f}}\} .
$$

These are commutative groups under the ideal multiplication. In particular, it is known that the quotient group

$$
C l(K, \widetilde{\mathfrak{f}})=A(\mathfrak{f}) / B(\widetilde{\mathfrak{f}})
$$

is a finite commutative group. We call it an ideal class group modulo $\tilde{\mathfrak{f}}$.

Let $\chi$ be a character of $C l(K, \widetilde{\mathfrak{f}})$. For $\Re s>1$, the $L$-function treated in this paper is defined as

$$
L(s, \chi)=\sum_{\mathfrak{a}} \frac{\chi(\mathfrak{a})}{N(\mathfrak{a})^{s}}
$$

where $\mathfrak{a}$ runs through all ideals of $O_{K}$ except 0 and $N(\mathfrak{a})$ denotes the norm of $\mathfrak{a}$.

Our result is as follows.

Theorem. Let $K$ be a finite extension of $\mathbb{Q}, n=[K: \mathbb{Q}]$ and $\chi$ be a character modulo $\widetilde{\mathfrak{f}}$. Set

$$
\sigma_{K}= \begin{cases}1 / 2 & \text { if } K=\mathbb{Q}, \\ 1-1 / n & \text { otherwise. }\end{cases}
$$

Let $D=D_{r}\left(s_{0}\right)=\left\{s \in \mathbb{C}|| s-s_{0} \mid \leq r\right\}$ be a disk contained in the strip $\sigma_{K}<\Re s<1$ and $f(s)$ be a continuous function on $D$ such that $f(s) \neq 0$ 
on $D$ and $f(s)$ is holomorphic in the interior of $D$. Then for every $\varepsilon>0$, we have

$$
\liminf _{T \rightarrow \infty} \frac{m\left(\left\{\tau \in[0, T]\left|\max _{s \in D}\right| L(s+i \tau, \chi)-f(s) \mid<\varepsilon\right\}\right)}{T}>0 .
$$

The organization of this paper is as follows. In Section 2 we state basic results on $L(s, \chi)$. In Section 3 we state sufficient conditions under which the universality theorem holds (Lemma 1) and show that in order to prove the theorem it is enough to prove a lemma which is connected with a Hardy space (Lemma 4). In Section 4 we prove that lemma by using arithmetic properties of $L(s, \chi)$. The key idea is to apply a result of class field theory (Lemma 6). In Section 5 we consider the case when $K$ is a Galois extension. In this case, we can prove our theorem by a more elementary method, without using class field theory.

REMARK. We prove the Theorem by the method based on Voronin's original proof. However we can also give a proof by a method based on Bagchi's alternative proof. In fact the conditions which are necessary to prove are almost the same.

The author would like to express his sincere gratitude to Professor Kohji Matsumoto and Professor Yoshio Tanigawa for their advice and encouragement. He would also like to express his sincere thanks to the referee for his helpful comments.

2. Background on $L$-functions. In this section, we recall fundamental properties of $L(s, \chi)$. See Mitsui [10] or [11] for details.

The $L$-function $L(s, \chi)$ can be meromorphically continued to the whole $s$-plane, and it is analytic except for a possible simple pole at $s=1$. In particular if $\chi$ is primitive, the function

$$
\xi(s, \chi)=D(\mathfrak{f})^{s} \Gamma(s)^{r_{2}} \prod_{m=1}^{r_{1}} \Gamma\left(\frac{s+a_{m}}{2}\right) L(s, \chi)
$$

satisfies the functional equation

$$
\xi(1-s, \bar{\chi})=\xi(s, \chi)
$$

where $r_{1}$ is the number of real places of $K, 2 r_{2}$ is the number of complex places of $K$, the constant $D(\mathfrak{f})$ depends only on $\mathfrak{f}$ and $a_{m}\left(1 \leq m \leq r_{1}\right)$, depending on $\chi$, takes the value 0 or 1 .

Hence by applying Potter's classical result [13], we have the estimate

$$
\int_{0}^{T}|L(\sigma+i t)| d t=O(T)
$$


for $\sigma>\sigma_{K}$. This estimate also holds for non-primitive characters $\chi$. In fact, if $\chi_{1}\left(\bmod \widetilde{\mathfrak{f}}_{1}\right)$ is a primitive character which induces $\chi$, then

$$
L(s, \chi)=L\left(s, \chi_{1}\right) \prod_{\mathfrak{p} \mid \mathfrak{f}}\left(1-\frac{\chi_{1}(\mathfrak{p})}{N(\mathfrak{p})^{s}}\right) .
$$

The finite product on the right-hand side is bounded, so we have the estimate (2) for every $\chi$.

The $L$-function $L(s, \chi)$ also satisfies the estimate

$$
L(s, \chi)(1-s)^{E(\chi)}=O\left((1+|t|)^{n+1}\right) \quad(1 / 2<\Re s<3 / 2)
$$

where

$$
E(\chi)= \begin{cases}1 & \text { if } \chi \text { is the identity } \\ 0 & \text { otherwise }\end{cases}
$$

and the $O$-constant depends only on $\chi$.

For $\Re s>1, L(s, \chi)$ satisfies the Euler product formula

$$
L(s, \chi)=\prod_{\mathfrak{p}}\left(1-\frac{\chi(\mathfrak{p})}{N(\mathfrak{p})^{s}}\right)^{-1}
$$

where $\mathfrak{p}$ runs through all prime ideals in $K$. We denote the decomposition of a prime number $p$ in $K$ as

$$
p=\mathfrak{p}_{1}^{x_{1}} \ldots \mathfrak{p}_{z_{p}}^{x_{z_{p}}}, \quad N\left(\mathfrak{p}_{i}\right)=p^{y_{i}} \quad\left(i=1, \ldots, z_{p}\right) .
$$

By using this expression, we have

$$
L(s, \chi)=\prod_{p}\left\{\prod_{i=1}^{z_{p}}\left(1-\frac{\chi\left(\mathfrak{p}_{i}\right)}{p^{y_{i} s}}\right)^{-1}\right\}
$$

so we set

$$
L(s, \chi)=\prod_{p} f_{p}\left(\frac{1}{p^{s}}\right), \quad f_{p}(z)=\prod_{i=1}^{z_{p}}\left(1-\chi\left(\mathfrak{p}_{i}\right) z^{y_{i}}\right)^{-1} .
$$

The functions $f_{p}(z)$ are clearly rational functions of $z$ and holomorphic in $|z|<1$ since $\left|\chi\left(\mathfrak{p}_{i}\right)\right|=1$. Writing the Taylor expansion of $f_{p}(z)$ as

$$
f_{p}(z)=1+\sum_{m=1}^{\infty} a_{p}^{(m)} z^{m}
$$

we have

$$
\begin{aligned}
\left|a_{p}^{(m)}\right| & =\left|\sum_{\substack{y_{1} j_{1}+\ldots+y_{z_{p}} j_{z_{p}}=m \\
j_{i} \geq 0}} \chi^{j_{i}}\left(\mathfrak{p}_{i}\right)\right| \leq \sum_{\substack{y_{1} j_{1}+\ldots+y_{z_{p}} j_{z_{p}}=m \\
j_{i} \geq 0}} 1 \\
& \leq(m+1)^{n} \ll_{\varepsilon} p^{m \varepsilon} .
\end{aligned}
$$


3. Lemmas. A. A. Karatsuba and S. M. Voronin [6] stated the conditions under which the universality theorems hold for functions given by infinite products over primes. First, we define some symbols.

Definition 1. (i) We set

$$
\Omega=\prod_{p} \mathbb{R}_{p}, \quad \mathbb{R}_{p}=\mathbb{R} .
$$

That is, $\Omega$ is the set of all real sequences indexed by primes.

(ii) Suppose that $F(s)$ is a meromorphic function in the strip $\sigma_{1}<\Re s<$ $\sigma_{2}$. If

$$
F(s)=O\left(|t|^{A}\right) \quad\left(|t|>T_{0}\left(\sigma_{1}^{\prime}, \sigma_{2}^{\prime}\right), \sigma_{1}^{\prime} \leq \Re s \leq \sigma_{2}^{\prime}\right)
$$

for any $\sigma_{1}^{\prime}, \sigma_{2}^{\prime}$ such that $\sigma_{1}<\sigma_{1}^{\prime}<\sigma_{2}^{\prime}<\sigma_{2}$, with some positive constant $A$ independent on $t$, then $F(s)$ is called a function of finite order.

(iii) Suppose that

$$
F(s)=\prod_{p} f_{p}\left(\frac{1}{p^{s}}\right)
$$

where $f_{p}(z)$ is a rational function of $z$. For a finite set of primes $M$ and $\bar{\theta}=\left(\theta_{p}\right) \in \Omega$, we define

$$
F_{M}(s, \bar{\theta})=\prod_{p \in M} f_{p}\left(\frac{e\left(-\theta_{p}\right)}{p^{s}}\right)
$$

where $e(x)=e^{2 \pi i x}$.

Lemma 1. Suppose that for $\Re s>1$ the analytic function $F(s)$ can be represented by the product

$$
F(s)=\prod_{p} f_{p}\left(\frac{1}{p^{s}}\right) .
$$

Suppose that $F(s)$ and $\left\{f_{p}(z)\right\}$ satisfy the following conditions.

(i) For every $p$, the function

$$
f_{p}(z)=1+\sum_{m=1}^{\infty} a_{p}^{(m)} z^{m}
$$

is a rational function of $z$ which has no poles in $|z|<1$ and has the property that for any $\varepsilon>0$

$$
\left|a_{p}^{(m)}\right|<c(\varepsilon) p^{m \varepsilon} \quad(m \in \mathbb{N})
$$

uniformly in $p$. 
(ii) $F(s)$ can be analytically continued onto the half-plane $\Re s>1 / 2$, with the possible exception of a finite number of poles on the line $\Re s=1$, has finite order in that half-plane and satisfies the estimate

$$
\frac{1}{T} \int_{-T}^{T}|F(\alpha+i t)|^{2} d t=O(1)
$$

for all $\alpha \in\left(\sigma_{0}, 1\right)$, where $\sigma_{0} \geq 1 / 2$.

(iii) There exist $\bar{\theta}=\left(\theta_{p}\right) \in \Omega$ and a sequence $M_{1} \subset M_{2} \subset \ldots$ of finite sets of prime numbers such that

(a) $\bigcup M_{j}$ contains all prime numbers,

(b) $F_{M_{j}}(s, \bar{\theta}) \rightarrow f(s)(j \rightarrow \infty)$ uniformly on $D=D_{r}\left(s_{0}\right)$ contained in the strip $\sigma_{0}<\Re s<1$.

Then for any $\varepsilon>0$, we have

$$
\liminf _{T \rightarrow \infty} \frac{m\left(\left\{\tau \in[0, T]\left|\max _{s \in D}\right| F(s+i \tau)-f(s) \mid<\varepsilon\right\}\right)}{T}>0 .
$$

To prove the Theorem, we apply Lemma 1 to $L(s, \chi)$ and $\left\{f_{p}(z)\right\}$ defined in Section 2. The conditions (i) and (ii) are clearly satisfied from (2)-(4) and the definition of $f_{p}(z)$. Hence it is enough to show that the condition (iii) also holds.

Since $f(s)$ is continuous on $D$, there exists $\gamma>1$ such that the disk $D_{r \gamma}\left(s_{0}\right)$ is contained in the strip $\sigma_{K}<\Re s<1$ and an inequality

$$
\max _{s \in D}\left|f(s)-f\left(\frac{s}{\gamma}\right)\right|<\varepsilon
$$

holds. Let $g(s)$ be an analytic function on $D$ such that

$$
f\left(\frac{s}{\gamma}\right)=e^{g(s)} \quad(s \in D) .
$$

Now we suppose that there exist a finite set $M$ of primes and $\bar{\theta} \in \Omega$ such that

$$
\max _{s \in D}\left|g(s)-\sum_{p \in M} \log f_{p}\left(\frac{e\left(-\theta_{p}\right)}{p^{s}}\right)\right|<\varepsilon .
$$

We note that, putting

$$
L_{M}(s, \chi, \bar{\theta})=\prod_{p \in M} f_{p}\left(\frac{e\left(-\theta_{p}\right)}{p^{s}}\right),
$$

we have 


$$
\begin{aligned}
\max _{s \in D}\left|f(s)-L_{M}(s, \chi, \bar{\theta})\right| & \\
& \leq \max _{s \in D}\left|f(s)-f\left(\frac{s}{\gamma}\right)\right|+\max _{s \in D}\left|e^{g(s)}-\exp \left\{\sum_{p \in M} \log f_{p}\left(\frac{e\left(-\theta_{p}\right)}{p^{s}}\right)\right\}\right| \\
& \ll \varepsilon+\max _{s \in D}\left|e^{g(s)}\right| \varepsilon \ll \varepsilon .
\end{aligned}
$$

Therefore the third condition in Lemma 1 follows from the following result.

LEMma 2. Let $g(s)$ be a continuous function on $D$ which is analytic in the interior of $D$. Then for every $\varepsilon>0$ and $y>0$, there exists a finite set $M$ of primes which satisfies the following conditions:

(i) $M$ contains all primes less than $y$,

(ii) we have

$$
\max _{s \in D}\left|g(s)-\sum_{p \in M} \log f_{p}\left(\frac{e\left(-\theta_{p}\right)}{p^{s}}\right)\right|<\varepsilon
$$

where $\bar{\theta}=\left(\theta_{p}\right) \in \Omega$, depending on $\mathfrak{f}$ and $D$, is defined below.

To prove Lemma 2, we introduce the notion of the Hardy space. First, we choose $\gamma>1$ such that the disk $D_{r \gamma}\left(s_{0}\right)$ is contained in the strip $\sigma_{K}<$ $\Re s<1$ and an inequality

$$
\max _{s \in D}\left|g(s)-g\left(\frac{s}{\gamma}\right)\right|<\varepsilon
$$

holds. Let $R=r \gamma$. The Hardy space on $D_{R}\left(s_{0}\right)$ is the set of all functions $f(s)$ which are analytic in the interior of $D_{R}\left(s_{0}\right)$ and

$$
\|f\|_{R}=\left(\iint_{D_{R}\left(s_{0}\right)}|f(s)|^{2} d \sigma d t\right)^{1 / 2}<\infty .
$$

We denote it by $H_{2}\left(D_{R}\left(s_{0}\right)\right)$. We define the inner product in $H_{2}\left(D_{R}\left(s_{0}\right)\right)$ by the formula

$$
(f, g)=\Re\left(\iint_{D_{R}\left(s_{0}\right)} f(s) \overline{g(s)} d \sigma d t\right) \quad\left(f, g \in H^{2}\left(D_{R}\left(s_{0}\right)\right)\right) .
$$

This makes $H_{2}\left(D_{R}\left(s_{0}\right)\right)$ into a real Hilbert space.

Now we define $\bar{\theta} \in \Omega$ as follows. Let $L / K$ be the class field on the ideal class group $C l(K, \widetilde{\mathfrak{f}})$, and $\mathbb{P}_{L}$ be the set of all rational primes which split completely in $L$. We will later show that $\mathbb{P}_{L}$ has infinite elements (see Lemma 7). We number primes in $\mathbb{P}_{L}$ in increasing order. If a prime $p$ appears as the $k$ th member of $\mathbb{P}_{L}$ in that order, then we define $l_{p}=k$.

We set

$$
\theta_{p}^{(0)}= \begin{cases}l_{p} / 4 & \text { if } p \in \mathbb{P}_{L} \\ 0 & \text { otherwise }\end{cases}
$$


and

$$
\theta_{p}=\theta_{p}^{(0)}-t_{0} \frac{\log p}{2 \pi}
$$

where $t_{0}$ is the imaginary part of $s_{0}=\sigma_{0}+i t_{0}$.

We note that $H_{2}\left(D_{R}\left(s_{0}\right)\right)$-norm convergence induces uniform convergence on compact subsets of $D_{R}\left(s_{0}\right)$. Namely, for $f(s) \in H_{2}\left(D_{R}\left(s_{0}\right)\right)$ we have

$$
\max _{\left|s-s_{0}\right| \leq r^{\prime}}|f(s)| \leq \frac{1}{\left(R-r^{\prime}\right) \sqrt{\pi}}\|f\|_{R}^{1 / 2} \quad\left(0<r^{\prime}<R\right)
$$

(see [6]). Using (5) and the fact that $g(s / \gamma)$ belongs to $H_{2}\left(D_{R}\left(s_{0}\right)\right)$, we can trivially deduce Lemma 2 from the following lemma.

Lemma 3. The set of all rearrangements of terms of the series

$$
\sum_{p} \log f_{p}\left(\frac{e\left(-\theta_{p}\right)}{p^{s}}\right)
$$

which converge in $H_{2}\left(D_{R}\left(s_{0}\right)\right)$ coincides with $H_{2}\left(D_{R}\left(s_{0}\right)\right)$ itself.

We prove Lemma 3 in the next section.

4. The proof of Lemma 3. The functions $f_{p}(z)$ themselves are not easy to deal with, hence we introduce functions which are easier. We set

$$
\alpha_{p}=\sum_{\substack{i=0 \\ y_{i}=1}}^{z_{p}} \chi\left(\mathfrak{p}_{i}\right), \quad h_{p}(s)=\frac{\alpha_{p} e\left(-\theta_{p}\right)}{p^{s}} .
$$

Then

$$
\begin{aligned}
& \sum_{p}\left\|h_{p}(s)-\log f_{p}\left(\frac{e\left(-\theta_{p}\right)}{p^{s}}\right)\right\|_{R} \\
& \quad=\sum_{p}\left(\iint_{D_{R}\left(s_{0}\right)}\left|\sum_{i=1}^{z_{p}} \sum_{k=1}^{\infty} \frac{\chi^{k}\left(\mathfrak{p}_{i}\right) e\left(-k y_{i} \theta_{p}\right)}{k p^{k y_{i} s}}-\sum_{\substack{i=1 \\
y_{i}=1}}^{z_{p}} \frac{\chi\left(\mathfrak{p}_{i}\right) e\left(-\theta_{p}\right)}{p^{s}}\right|^{2} d \sigma d t\right)^{1 / 2} \\
& \quad \ll \sum_{p} \sum_{k=2}^{\infty} \frac{1}{k p^{k\left(\sigma_{0}-R\right)}}+\sum_{p} \sum_{k=1}^{\infty} \frac{1}{k p^{2 k\left(\sigma_{0}-R\right)}}<\infty
\end{aligned}
$$

since $\sigma_{0}-R>1 / 2$. Hence it is enough to prove Lemma 3 for the series $\sum_{p} h_{p}(s)$ instead of the series $\sum_{p} \log f_{p}\left(e\left(-\theta_{p}\right) / p^{s}\right)$.

Now recall a result of Pecherskilu.

Lemma 4 (Pecherskiı [12]). Let $H$ be a real Hilbert space. Suppose that $\left\{u_{n}\right\} \subset H$ satisfies the following conditions.

(i) $\sum_{n=1}^{\infty}\left\|u_{n}\right\|^{2}<\infty$. 
(ii) For any $e \in H$ with $\|e\|=1$, the series $\sum_{n=1}^{\infty}\left(u_{n}, e\right)$ is not absolutely convergent but conditionally convergent for some arrangement of the terms.

Then the set of all rearrangements of terms of the series $\sum_{n=1}^{\infty} u_{n}$ which converge in $H$ coincides with $H$ itself.

We note that $H_{2}\left(D_{R}\left(s_{0}\right)\right)$ is a real Hilbert space and $h_{p}(s) \in H_{2}\left(D_{R}\left(s_{0}\right)\right)$. Hence it is sufficient to show that $\left\{h_{p}(s)\right\}$ satisfies two conditions in Lemma 4.

We have

$$
\sum_{p}\left\|h_{p}(s)\right\|_{R}^{2}=\sum_{p} \iint_{D_{R}\left(s_{0}\right)}\left|\frac{\alpha_{p} e\left(-\theta_{p}\right)}{p^{s}}\right|^{2} d \sigma d t \ll \sum_{p} \frac{1}{p^{2\left(\sigma_{0}-R\right)}}<\infty
$$

since $\sigma_{0}-R>1 / 2$. Hence $h_{p}(s)$ satisfies the first condition.

Next we show that, for any $\phi(s) \in H_{2}\left(D_{R}\left(s_{0}\right)\right)$ with $\|\phi\|=1$, the series

$$
\sum_{p}\left(h_{p}, \phi\right)
$$

is not absolutely convergent but conditionally convergent for some arrangement of terms. To do this, it is enough to show that

- $\left(h_{p}, \phi\right) \rightarrow 0(p \rightarrow \infty)$.

- There exist two subseries of $\sum_{p}\left(h_{p}, \phi\right)$, one diverging to $+\infty$ and the other to $-\infty$.

The first condition holds since

$$
\left|\left(h_{p}, \phi\right)\right| \leq\left\|h_{p}\right\| \cdot\|\phi\|=\iint_{D_{R}\left(s_{0}\right)} \frac{\left|\alpha_{p}\right|^{2}}{p^{2 \sigma}} d \sigma d t \ll \frac{1}{p^{2\left(\sigma_{0}-R\right)}} \rightarrow 0 \quad(p \rightarrow \infty) .
$$

Next, we set

$$
\phi(s)=\sum_{m=0}^{\infty} a_{m}\left(s-s_{0}\right)^{m} .
$$

We express $\left(h_{p}, \phi\right)$ in terms of $a_{m}$ :

$$
\begin{aligned}
& \left(h_{p}, \phi\right)=\Re\left(\iint_{D_{R}\left(s_{0}\right)} \frac{\alpha_{p} e\left(-\theta_{p}\right)}{p^{s}} \overline{\phi(s)} d \sigma d t\right) \\
& =\Re\left(\frac{\alpha_{p} e\left(-\theta_{p}\right)}{p^{s_{0}}} \iint_{D_{R}\left(s_{0}\right)} e^{-\left(s-s_{0}\right) \log p}\left(\sum_{m=0}^{\infty} \bar{a}_{m}\left(\overline{s-s_{0}}\right)^{m}\right) d \sigma d t\right) \\
& =\Re\left(\frac{\alpha_{p} e\left(-\theta_{p}\right)}{p^{s_{0}}} \iint_{|s| \leq R}\left(\sum_{k=0}^{\infty} \frac{(-s \log p)^{k}}{k !}\right)\left(\sum_{m=0}^{\infty} \bar{a}_{m} \bar{s}^{m}\right) d \sigma d t\right) \\
& =\Re\left(\frac{\alpha_{p} e\left(-\theta_{p}\right)}{p^{s_{0}}} \sum_{k=0}^{\infty} \sum_{m=0}^{\infty} \frac{(-\log p)^{k} \bar{a}_{m}}{k !} \iint_{|s| \leq R} s^{k} \bar{s}^{m} d \sigma d t\right) .
\end{aligned}
$$


Passing to polar coordinates, we have

$$
\iint_{|s| \leq R} s^{k} \bar{s}^{m} d \sigma d t=\int_{0}^{R} \int_{0}^{2 \pi} r^{k+m+1} e^{i(k-m) \theta} d r d \theta= \begin{cases}\frac{\pi R^{2 m+2}}{m+1} & (m=k), \\ 0 & (m \neq k) .\end{cases}
$$

Hence

$$
\left(h_{p}, \phi\right)=\Re\left(\frac{\alpha_{p} e\left(-\theta_{p}\right)}{p^{s_{0}}} G(R \log p)\right)
$$

where

$$
b_{m}=\frac{(-1)^{m} \pi \bar{a}_{m} R^{m+2}}{m+1}, \quad G(x)=\sum_{m=0}^{\infty} \frac{b_{m}}{m !} z^{m} .
$$

Now we consider $G(x)$. Since $\|\phi\|=1$, we have

$$
1=\|\phi\|^{2}=\iint_{D_{R}\left(s_{0}\right)}\left|\sum_{m=0}^{\infty} a_{m}\left(s-s_{0}\right)^{m}\right|^{2} d \sigma d t=\sum_{m=0}^{\infty} \frac{\pi R^{2 m+2}}{m+1}\left|a_{m}\right|^{2} .
$$

Hence $0<\sum_{m=0}^{\infty}\left|b_{m}\right|^{2} \leq 1$. In particular $\left|b_{m}\right| \leq 1$ for all $m \leq 0$. Hence $G(x)$ is an entire function which is not 0 identically. In particular, we have the following lemma.

LEMMA 5. Let

$$
F(x)=\sum_{m=0}^{\infty} \frac{b_{m}}{m !} x^{m}
$$

with $\left|b_{m}\right| \leq 1$ for all $m \geq 0$. Then there exist a real sequence $\left\{R_{m}\right\}$ which tends to $+\infty$ and positive constants $c_{1}, c_{2}$ such that if we set $l_{m}=c_{1} /\left(R_{m}+2\right)$ and $I_{m}=\left[R_{m}, R_{m}+l_{m}\right]$ then

$$
|\Re G(x)|>c_{2} / e^{x} \quad \text { for any } x \in I_{m} .
$$

If the character sums

$$
\alpha_{p}=\sum_{\substack{i=1 \\ y_{i}=1}}^{z_{p}} \chi\left(\mathfrak{p}_{i}\right)
$$

were 0 for almost all $p$, then we could not prove the existence of desired subseries. However, such a situation never happens. We can show that as follows.

Recall that $L / K$ is the class field on the ideal class group $C l(K, \widetilde{\mathfrak{f}})$. Then from Takagi [15] or Hasse [5], we have

Lemma 6. Let $\mathfrak{p}$ be a prime ideal in $K$. The following two conditions are equivalent:

(i) $\mathfrak{p}$ completely splits in $L$,

(ii) $\mathfrak{p} \in B(\widetilde{\mathfrak{f}})$ where $B(\widetilde{\mathfrak{f}})$ is the ideal group defined by (1). 
Let $p \in \mathbb{P}_{L}$. Then $p$ completely splits in $K$ as $p=\mathfrak{p}_{1} \ldots \mathfrak{p}_{n}$ and each $\mathfrak{p}_{i}$ completely splits in $L$. Hence by Lemma 6 , we have

$$
\chi\left(\mathfrak{p}_{i}\right)=1 \quad(i=0, \ldots, n) .
$$

Hence for any $p \in \mathbb{P}_{L}$ we have $\alpha_{p}=n$.

By applying the above results, we now prove existence of a subseries diverging to $+\infty$. We apply Lemma 5 to $G(x)$ defined by (7). We define finite sets $K_{m}(m \in \mathbb{N})$ of primes as follows.

- If $\Re(G(x))>c_{2} / e^{x}\left(x \in I_{m}\right)$, then

$$
K_{m}=\left\{p \mid p \in \mathbb{P}_{L}, R \log p \in I_{m}, l_{p} \equiv 0(\bmod 4)\right\} .
$$

- If $\Re(G(x))<-c_{2} / e^{x}\left(x \in I_{m}\right)$, then

$$
K_{m}=\left\{p \mid p \in \mathbb{P}_{L}, R \log p \in I_{m}, l_{p} \equiv 2(\bmod 4)\right\},
$$

where $l_{p}$ is defined in Section 3 .

For every $p \in K_{m}$, by the definition (6) of $\theta_{p}$, Lemma 5 and the fact that $\alpha_{p}=n$ for any $p \in \mathbb{P}_{L}$, we have

$$
\begin{aligned}
\left(h_{p}, \phi\right) & =\Re\left(\frac{\alpha_{p} e\left(-\theta_{p}\right)}{p^{s_{0}}} G(R \log p)\right)=\frac{n}{p^{\sigma_{0}}}|\Re(G(R \log p))| \\
& \gg \exp \left(-\left(\frac{\sigma_{0}}{R}+1\right)\left(R_{m}+l_{m}\right)\right) .
\end{aligned}
$$

On the other hand, the number of elements of $K_{m}$ is

$$
\sharp K_{m}=\frac{1}{4}\left\{\pi\left(e^{\left(R_{m}+l_{m}\right) / R}, L\right)-\pi\left(e^{R_{m} / R}, L\right)\right\}+o(1)
$$

by the definition of $K_{m}$, where we define

$$
\pi(x, L)=\sum_{\substack{p \leq x \\ p \in \mathbb{P}_{L}}} 1 .
$$

Let $\bar{L}$ be the Galois closure of $L$. We can show by algebraic methods that, for $p$ unramified in $L, p$ splits completely in $L$ if and only if $p$ splits completely in $\bar{L}$. Also we note that there exist only finitely many primes which ramify in $L$. Hence

$$
\pi(x, L)=\pi(x, \bar{L})+O(1) .
$$

We apply the following result.

Lemma 7 (Artin [1]). Let $F / k$ be a Galois extension, $G=\operatorname{Gal}(F / k)$, and $A$ be a conjugacy class of $G$. Set $\pi(x, A)=\sharp\{\mathfrak{p}:$ prime ideal in $k \mid N(\mathfrak{p}) \leq x$, Frobenius class of $\mathfrak{p}$ is $A\}$. 
Then

$$
\pi(x, A)=\frac{\sharp A}{\sharp G} \int_{2}^{x} \frac{d t}{\log t}+O\left(x e^{-c \sqrt{\log x}}\right) .
$$

Since $\bar{L} / \mathbb{Q}$ is a Galois extension, and a prime $p$ completely splits in $L$ if and only if the Frobenius class of $p$ is $\{1\}$, by Lemma 7 we have

$$
\pi(x, L)=\frac{1}{N_{L}} \int_{2}^{x} \frac{d t}{\log x}+O\left(x e^{-c \sqrt{\log x}}\right)
$$

where $N_{L}=[\bar{L}: \mathbb{Q}]$. By using this formula, we obtain

$$
\begin{aligned}
\sharp K_{m}= & \frac{1}{4 N_{L}}\left\{\int_{e^{R m / R}}^{e^{\left(R_{m}+l_{m}\right) / R}} \frac{d t}{\log t}+O\left(e^{\left(R_{m}+l_{m}\right) / R} e^{-c \sqrt{\left(R_{m}+l_{m}\right) / R}}\right)\right\} \\
= & \frac{1}{4 N_{L}} e^{R_{m} / R}\left\{\frac{R_{m}\left(e^{l_{m} / R}-1\right)-l_{m}}{R_{m}\left(R_{m}+l_{m}\right)}+O\left(\frac{e^{l_{m} / R}-1}{R_{m}^{2}}\right)\right. \\
& \left.+O\left(e^{R_{m} / R} e^{-c \sqrt{\left(R_{m}+l_{m}\right) / R}}\right)\right\} .
\end{aligned}
$$

Since $l_{m}=c_{1} /\left(R_{m}+2\right)$, we have

$$
\sharp K_{m} \gg e^{R_{m} / R} / R_{m}^{3} \text {. }
$$

Then (8) and (9) imply

$$
\sum_{p \in K_{m}}\left(h_{p}, \phi\right) \gg \frac{1}{R_{m}^{3}} \exp \left(\left(\frac{1-\sigma_{0}}{R}-1\right) R_{m}\right) \exp \left(-\left(\frac{\sigma_{0}}{R}+1\right) l_{m}\right) .
$$

Since $\sigma_{0}+R<1$, we have $\left(1-\sigma_{0}\right) / R-1>0$. Hence the second factor on the right-hand side diverges to $+\infty$ exponentially as $m \rightarrow \infty$. And the third factor converges to 1 since $l_{m}$ converges to 0 as $m \rightarrow \infty$. Hence we conclude that there exists a subseries diverging to $+\infty$.

To prove that there exist subseries diverging to $-\infty$, we define $K_{m}$ as follows.

- If $\Re(G(x))>c_{2} / e^{x}\left(x \in I_{m}\right)$, then

$$
K_{m}=\left\{p \mid p \in \mathbb{P}_{L}, R \log p \in I_{m}, l_{p} \equiv 2(\bmod 4)\right\} .
$$

- If $\Re(G(x))<-c_{2} / e^{x}\left(x \in I_{m}\right)$, then

$$
K_{m}=\left\{p \mid p \in \mathbb{P}_{L}, R \log p \in I_{m}, l_{p} \equiv 0(\bmod 4)\right\} .
$$

Then

$$
\sum_{p \in K_{m}}\left(h_{p}, \phi\right) \ll-\frac{1}{R_{m}^{3}} \exp \left(\left(\frac{1-\sigma_{0}}{R}-1\right) R_{m}\right) \exp \left(-\left(\frac{\sigma_{0}}{R}+1\right) l_{m}\right) .
$$

From this estimate, we infer that there exists a subseries diverging to $-\infty$. 
5. The case when $K / \mathbb{Q}$ is Galois. The key to our proof of the universality theorem is the consideration of the character sums

$$
\alpha_{p}=\sum_{\substack{i=1 \\ y_{i}=1}}^{z_{p}} \chi\left(\mathfrak{p}_{i}\right) .
$$

To show that there exist many primes such that $\alpha_{p} \neq 0$, we apply class field theory. However if $K / \mathbb{Q}$ is Galois, we can prove our theorem by a more elementary method. We use the same symbols as in the previous section unless otherwise indicated.

Let $K / \mathbb{Q}$ be a Galois extension of degree $n$. The decomposition of a prime number $p$ in $K$ is

$p=\left(\mathfrak{p}_{1} \ldots \mathfrak{p}_{z_{p}}\right)^{x_{p}}, N\left(\mathfrak{p}_{i}\right)=p^{y_{p}}\left(i=1, \ldots, z_{p}\right), \quad x_{p} y_{p} z_{p}=n, x_{p}, y_{p}, z_{p} \in \mathbb{N}$

where we note that all $\mathfrak{p}_{i}$ are conjugate to each other.

Now we define $f=N(\mathfrak{f})$ and an ideal class group

$$
B((\widetilde{f}))=\{(\alpha) \mid \alpha \in K, \alpha \equiv 1(\bmod (\widetilde{f}))\} .
$$

Let $\mathfrak{a}=(\alpha) \in B((\widetilde{f}))$. We recall that $\alpha \equiv 1(\bmod (\widetilde{f}))$ if and only if

$$
\alpha=b / a, \quad a, b \in O_{K}, a-b \in(f), \quad \alpha \succ 0 .
$$

Let $\sigma \in G=\operatorname{Gal}(K / \mathbb{Q})$. Then $\mathfrak{a}^{\sigma}=\left(\alpha^{\sigma}\right)$. Since $f \in \mathbb{N}$, we have

$$
\alpha^{\sigma}=a^{\sigma} / b^{\sigma}, \quad a^{\sigma}-b^{\sigma} \in(f),
$$

and $\alpha^{\sigma}$ is clearly totally positive. Hence if $\mathfrak{a} \in B((\widetilde{f}))$ then for any $\sigma \in G$,

$$
\mathfrak{a}^{\sigma} \in B((\widetilde{f})) \text {. }
$$

Therefore for $p=\left(\mathfrak{p}_{1} \ldots \mathfrak{p}_{z_{p}}\right)^{x_{p}}$, if $\mathfrak{p}_{1} \in B((\tilde{f}))$ then all $\mathfrak{p}_{i}\left(i=1, \ldots, z_{p}\right)$ are included in $B((\widetilde{f}))$. Since $\mathfrak{f} \mid(f), B((\widetilde{f}))$ is a subgroup of $B(\widetilde{\mathfrak{f}})$. Hence letting $\mathbb{P}_{(f)}$ be the set of primes $p$ which split completely in $K$ such that $\mathfrak{p}_{1} \in B((\widetilde{f}))$, for any $p \in \mathbb{P}_{(f)}$ we have

$$
\alpha_{p}=n .
$$

Next we define $\bar{\theta} \in \Omega$ as follows. We will later show that $\mathbb{P}_{(f)}$ has infinitely many elements (see Lemma 8). We number the primes in $\mathbb{P}_{(f)}$ in increasing order. If a prime $p$ appears as the $k$ th member of $\mathbb{P}_{(f)}$ in that order, we define $l_{p}=k$. We set

$$
\theta_{p}^{(0)}= \begin{cases}l_{p} / 4 & \text { if } p \in \mathbb{P}_{(f)}, \\ 0 & \text { otherwise }\end{cases}
$$

and

$$
\theta_{p}=\theta_{p}^{(0)}-t_{0} \frac{\log p}{2 \pi}
$$


Now, for a prime $p$ which splits completely in $K$, we set

$$
h_{p}(s)=\frac{\alpha_{p} e\left(-\theta_{p}\right)}{p^{s}} \text {. }
$$

As in Section 4, we see that it is enough to prove that, for any $\phi \in H_{2}\left(D_{R}\left(s_{0}\right)\right)$ with $\|\phi\|_{R}=1$, there exist two subseries of the series $\sum_{p}\left(h_{p}, \phi\right)$, one diverging to $+\infty$ and the other to $-\infty$.

We construct a subseries diverging to $+\infty$ as follows. We take intervals $I_{m}(m \in \mathbb{N})$ as in Lemma 5 , and define finite sets $K_{m}(m \in \mathbb{N})$ of primes as follows.

- If $\Re(G(x))>c_{2} / e^{x}\left(x \in I_{m}\right)$, then

$$
K_{m}=\left\{p \mid p \in \mathbb{P}_{(f)}, R \log p \in I_{m}, l_{p} \equiv 2(\bmod 4)\right\} .
$$

- If $\Re(G(x))<-c_{2} / e^{x}\left(x \in I_{m}\right)$, then

$$
K_{m}=\left\{p \mid p \in \mathbb{P}_{(f)}, R \log p \in I_{m}, l_{p} \equiv 0(\bmod 4)\right\} .
$$

For $p \in K_{m}$, by the definition (10) of $\theta_{p}$, Lemma 5 and the fact that $\alpha_{p}=n$ for any $p \in \mathbb{P}_{(f)}$, we have

$$
\begin{aligned}
\left(h_{p}, \phi\right) & =\Re\left(\frac{\alpha_{p} e\left(-\theta_{p}\right)}{p^{s_{0}}} G(R \log p)\right)=\frac{n}{p^{\sigma_{0}}}|\Re(G(R \log p))| \\
& \gg \exp \left(-\left(\frac{\sigma_{0}}{R}+1\right)\left(R_{m}+l_{m}\right)\right) .
\end{aligned}
$$

On the other hand, the cardinality of $K_{m}$ is

$$
\sharp K_{m}=\frac{1}{4}\left\{\pi\left(e^{\left(R_{m}+l_{m}\right) / R}, \mathbb{P}_{(f)}\right)-\pi\left(e^{R_{m} / R}, \mathbb{P}_{(f)}\right)\right\}+O(1)
$$

by the definition of $K_{m}$, where we set

$$
\pi\left(x, \mathbb{P}_{(f)}\right)=\sum_{\substack{p \leq x \\ p \in \mathbb{P}(f)}} 1 .
$$

We apply the following result of Mitsui [11].

Lemma 8. Let $k / \mathbb{Q}$ be a Galois extension, $C l(k, \widetilde{\mathfrak{a}})$ the ideal group of $k$ modulo $\widetilde{\mathfrak{a}}$ and $C$ an ideal class in $C l(k, \widetilde{\mathfrak{a}})$. Set

$$
\pi(x, C)=\sharp\{\mathfrak{p}: \text { prime ideal in } k \mid N(\mathfrak{p}) \leq x, \mathfrak{p} \in C\} \text {. }
$$

Then

$$
\pi(x, C)=\frac{1}{h(\widetilde{\mathfrak{a}})} \int_{2}^{x} \frac{d t}{\log t}+O\left(x e^{-c \sqrt{\log x}}\right)
$$

where $h(\widetilde{\mathfrak{a}})$ is the order of $C l(k, \widetilde{\mathfrak{a}})$. 
We note that $B((\widetilde{f}))$ is a principal class in $C l(k, \widetilde{a})$. Hence by Lemma 8 we have

$$
\sum_{\substack{N \mathfrak{p} \leq x \\ \mathfrak{p} \in B((\tilde{f}))}} 1=\frac{1}{h((\widetilde{f}))} \int_{2}^{x} \frac{d t}{\log t}+O\left(x e^{-c \sqrt{\log x}}\right) .
$$

The left-hand side is

$$
\sum_{\substack{N \mathfrak{p} \leq x \\ \mathfrak{p} \in B((\tilde{f}))}} 1=\sum_{\substack{N \mathfrak{p} \leq x \\ \mathfrak{p} \in B((\tilde{f})), N \mathfrak{p}=p}} 1+\sum_{\substack{N \mathfrak{p} \leq x \\ \mathfrak{p} \in B((\tilde{f})), N \mathfrak{p}=p^{y}}} 1 .
$$

The second term on the right is

$$
\ll \sum_{p^{2} \leq x} 1+\sum_{p^{3} \leq x} 1+\ldots+\sum_{p^{n} \leq x} 1=O\left(x^{1 / 2}\right)=O\left(x e^{-c \sqrt{\log x}}\right) .
$$

And a prime number $p$ splitting completely in $K$ corresponds to $n$ prime ideals which are conjugate to each other. Hence

$$
\pi\left(x, \mathbb{P}_{(f)}\right)=\frac{1}{n h((\widetilde{f}))} \int_{2}^{x} \frac{d t}{\log t}+O\left(x e^{-c \sqrt{\log x}}\right) .
$$

By using this formula, we calculate $\sharp K_{m}$ just as for (9), to obtain

$$
\sharp K_{m} \gg e^{R_{m} / R} / R_{m}^{3} \text {. }
$$

By (11) and (12), we have

$$
\sum_{p \in K_{m}}\left(h_{p}, \phi\right) \gg \frac{1}{R_{m}^{3}} \exp \left(\left(\frac{1-\sigma_{0}}{R}-1\right) R_{m}\right) \exp \left(-\left(\frac{\sigma_{0}}{R}+1\right) l_{m}\right) .
$$

As in the previous section, we can prove that there exists a subseries diverging to $+\infty$.

\section{References}

[1] E. Artin, Über eine neue Art von L-Reihen, Abh. Math. Sem. Univ. Hamburg (1923), 105-124.

[2] B. Bagchi, The statistical behaviour and universality properties of the Riemann zetafunction and other allied Dirichlet series, Ph.D. thesis, Indian Statistical Institute, Calcutta, 1981.

[3] -, A joint universality theorem for Dirichlet series, Math. Z. 181 (1982), 319-334.

[4] S. M. Gonek, Analytic properties of zeta and L-functions, Ph.D. thesis, University of Michigan, 1979.

[5] H. Hasse, Vorlesungen über Klassenkörpertheorie, Physica-Verlag, Würzburg, 1967.

[6] A. A. Karatsuba and S. M. Voronin, The Riemann Zeta-Function, de Gruyter, 1992.

[7] A. Laurinčikas, On the Matsumoto zeta-function, Acta Arith. 84 (1998), 1-16.

[8] A. Laurinčikas and K. Matsumoto, The universality of the zeta-functions attached to certain cusp forms, this issue, 345-359. 
[9] K. Matsumoto, Value-distribution of zeta functions, in: Lecture Notes in Math. 1434, Springer, 1990, 178-187.

[10] T. Mitsui, Kaisekitekisuuron, Iwanami, 1989 (in Japanese).

[11] -, Generalized prime number theorem, Japan J. Math. 26 (1956), 1-42.

[12] D. V. Pecherskiı̌, On rearrangements of terms in functional series, Soviet Math. Dokl. 14 (1973), 633-636.

[13] H. S. A. Potter, The mean values of certain Dirichlet series 1, Proc. London Math. Soc. 46 (1940), 467-478.

[14] A. Reich, Werteverteilung von Zetafunktionen, Arch. Math. (Basel) 34 (1980), 440451.

[15] T. Takagi, Daisuutekiseisuuron, Iwanami, 1970 (in Japanese).

[16] S. M. Voronin, A theorem on the "universality" of the Riemann zeta-function, Izv. Akad. Nauk SSSR Ser. Mat. 39 (1975), 475-486 (in Russian); English transl.: Math. USSR-Izv. 9 (1975), 443-453.

Graduate School of Mathematics

Nagoya University

Ghikusa-ku, Nagoya 464-8602, Japan

E-mail: m98018a@math.nagoya-u.ac.jp

Received on 16.5.2000

and in revised form on 3.8.2000 